\title{
c-Jun N-terminal kinase has a key role in Alzheimer disease synaptic dysfunction in vivo
}

\author{
A Sclip ${ }^{1}$, A Tozzi ${ }^{2,3}$, A Abaza ${ }^{4}$, D Cardinetti ${ }^{1}$, I Colombo ${ }^{1}$, P Calabresi ${ }^{2,3}$, M Salmona ${ }^{1}$, E Welker ${ }^{4}$ and T Borsello ${ }^{*, 1}$
}

Altered synaptic function is considered one of the first features of Alzheimer disease (AD). Currently, no treatment is available to prevent the dysfunction of excitatory synapses in AD. Identification of the key modulators of synaptopathy is of particular significance in the treatment of AD. We here characterized the pathways leading to synaptopathy in TgCRND8 mice and showed that c-Jun N-terminal kinase (JNK) is activated at the spine prior to the onset of cognitive impairment. The specific inhibition of JNK, with its specific inhibiting peptide D-JNKI1, prevented synaptic dysfunction in TgCRND8 mice. D-JNKI1 avoided both the loss of postsynaptic proteins and glutamate receptors from the postsynaptic density and the reduction in size of excitatory synapses, reverting their dysfunction. This set of data reveals that JNK is a key signaling pathway in AD synaptic injury and that its specific inhibition offers an innovative therapeutic strategy to prevent spine degeneration in AD.

Cell Death and Disease (2014) 5, e1019; doi:10.1038/cddis.2013.559; published online 23 January 2014

Subject Category: Neuroscience

Alzheimer disease $(A D)$ is characterized by loss of memory and cognition and ultimately by massive neuronal death. Substantial synaptic dysfunction is detected in the early stages of $A D$ when the hippocampus-dependent memory deficit becomes clinically detectable. ${ }^{1-3}$

Evidence demonstrates that soluble $A \beta$ oligomers interfered with the function of the excitatory synapses ${ }^{4-7}$ and induced removal of glutamate receptors from the postsynaptic density (PSD), leading to synaptopathy. ${ }^{8-15}$ Both $\mathrm{N}$-methyl D-aspartate receptors (NMDAr) ${ }^{16-20}$ and amino-3-hydroxy-5methyl-4isoxazole receptors (AMPAr) ${ }^{21,22}$ are affected. The reduction of glutamate receptors correlates with the drop in the synaptic levels of PSD-95, a postsynaptic scaffold protein regulating the recruitment and maintenance of both AMPAr and NMDAr within the postsynaptic membrane ${ }^{21,23}$ Functionally, $\mathrm{A} \beta$ oligomers affect long-term potentiation (LTP $)^{24}$ and long-term depression (LTD) ${ }^{25}$ by modulating glutamate receptor and trigger aberrant patterns of neural network activity. ${ }^{26}$

Although it's now clear that synaptic loss correlates with $A D$ cognitive impairments, the intracellular mechanisms leading to synaptic dysfunction/dysmorphogenesis remain largely unexplained. Understanding the pathological mechanisms is thus necessary to develop therapeutic approaches aimed at protecting against synaptopathy or at restoring its effect.

Because synaptic injury precedes neuronal death and surviving neurons possess a remarkable capacity for synaptic repair and functional recovery, we focus our efforts on the development of a strategy to protect synapses.
We here characterized the early events leading to synaptopathy in the hippocampus of TgCNRD8 mice, which manifested the first cognitive defects at 3 months of age. ${ }^{27}$ As JNK's role in synaptopathy has not yet been explored, we combined detailed biochemical studies on the PSD with morphological analyses and electrophysiological recordings to unveil the central role of $\mathrm{JNK}$ in the mechanisms leading to synaptic dysfunction.

We showed that JNK controls the first signs of spine alterations in the brain and that its inhibition protects against degeneration of dendritic spines in vivo. Our study sets the basis for a novel target in $A D$ therapy with particular regard to early synaptic dysfunction.

\section{Results}

Characterization of synaptopathy in TgCRND8 mice. To characterize the synaptic dysfunction in TgCRND8 mice, we isolated the postsynaptic elements using a well-established biochemical approach. ${ }^{28}$ The triton insoluble fraction (TIF) enriched in postsynaptic proteins was extracted from hippocampi of TgCRND8 mice and age-matched wild-type mice. To verify whether synaptic dysfunction occurred before the onset of cognitive deficits in this mouse strain, ${ }^{27}$ samples from 2-months-old mice were used. At this stage TgCRND8 mice already showed significantly reduced levels of GluN2A (31\%) and GluN2B (54\%) subunits of NMDAr; GluA1 (46\%) and GluA2 (35\%) subunits of AMPAr and a loss of postsynaptic markers like PSD-95 (35\%) and drebrin (60\%) (Student's $t$-test, $P<0.05)$ (Figures $1 \mathrm{a}-\mathrm{h}$ ).

\footnotetext{
${ }^{1}$ IRCCS-Istituto di Ricerche Farmacologiche 'Mario Negri', Via La Masa 19, 20156 Milano, Italy; ${ }^{2}$ Clinica Neurologica, Università di Perugia, Ospedale S. Maria della Misericordia, S. Andrea delle Fratte, 06156 Perugia, Italy; ${ }^{3}$ IRCCS-Fondazione S. Lucia, 00179 Roma, Italy and ${ }^{4}$ Département de Neurosciences Fondamentales, University of Lausanne, 1005 Lausanne, Switzerland

*Corresponding author: T Borsello, Neuronal Death and Neuroprotection Laboratory, IRCCS-Istituto Di Ricerche Farmacologiche 'Mario Negri', Via la Masa 19,20156 Milano, Italy. Tel: +39 0239014 469; Fax: +39 023900 1916; E-mail: tiziana.borsello@marionegri.it

Keywords: synaptic dysfunction; soluble A $\beta$ oligomers; signal transduction; therapeutics; cell permeable peptide; D-JNKI1

Abbreviation: AD, Alzheimer disease; AMPAr, amino-3-hydroxy-5-methyl-4isoxazole receptor; JNK, c-Jun N-terminal kinase; NMDAr, N-methyl D-aspartate receptor; PSD, postsynaptic density; TIF, triton insoluble fraction

Received 26.11.13; accepted 16.12.13; Edited by A Verkhratsky
} 
At 9 months of age, TgCRND8 presented a significant drop of glutamate receptor subunits: GluN2A (74\%), GluN2B (86\%), GluA1 (66\%) and GluA2 (71\%) (Figures 1i-m) (Student's t-test, $P<0.05)$. Similarly PSD-95 (80\%) and drebrin (84\%) were considerably reduced if compared with age-matched wild-type mice (Figures $1 \mathrm{n}$ and o) (Student's $t$-test, $P<0.05$ ).

We thus proved that synaptopathy started (2 months) altering the biochemical composition of the PSD before the manifestation of cognitive impairment (3 months) and the severity of PSD changes increased with age in TgCRND8 mice.

The JNK signaling pathway in synaptopathy. We then analyzed JNK activation at the postsynaptic level in TgCRND8 mice at 2 months, just before the manifestation of the cognitive impairment and at 9 months of age, when mice were severely affected. The western blot analyses demonstrated a significant activation of the JNK pathway in the postsynaptic compartment, already at 2 months of age and this correlated with the first biochemical changes in the PSD region of dendritic spines. The activated form of JNK was increased already in the pre-symptomatic stage (1.87fold, Figures $2 \mathrm{a}$ and $\mathrm{b}$ ) (Student's $t$-test, $P<0.05)$, and was further augmented in 9-months-old TgCRND8 mice (2.25fold; Figures 2c and d) (Student's $t$-test, $P<0.01$ ) in comparison with values obtained from age-matched, wildtype mice. These results indicated that JNK is implicated in the early phase of synaptic alterations, as its activation coincides with the first biochemical changes at the synaptic level and occurs before the manifestation of cognitive deficits. Importantly, JNK activation persisted and increased in time during synaptopathy progression in TgCRND8 mice.

Specific JNK inhibition prevents biochemical changes of the PSD region. To clarify the role of JNK in synaptopathy, we chronically treated TgCRND8 mice to with the specific JNK inhibitor peptide D-JNKI1 in order to prevent JNK action. Vehicle-treated mice were used as controls. Control peptides (TAT-empty and TAT-mutated peptides) were already tested in the same model and resulted to have any effect at the biochemical as well as at the electrophysiological levels. ${ }^{29}$ The treatment was started at the 4th month of age when the mice already showed cognitive decline $^{29}$ as well as biochemical changes in the postsynaptic element (Figures 1a-h). Wild-type control mice were also used for the treatment and received either the vehicle or D-JNKI1. After 5 months of treatment, hippocampi from wild type (wt) and TgCRND8 mice were dissected, processed for PSD extraction and analyzed by western blot.

D-JNKI1-treated TgCRND8 mice showed a significant increase in the levels of GluN2A, GluN2B, GluA1, GluA2, PSD-95 and drebrin in comparison with the levels from TgCRND8 mice treated with the vehicle (Figure 3a-h) (Twoway ANOVA, interaction $P<0.05$, Bonferroni post-hoc test). D-JNKI1 completely prevented alterations in the PSD composition since protein levels were similar to age-matched wt animals. D-JNKI1-treated wt mice did not present any change in PSD proteins (Figure 3a). As expected, D-JNKI1 treatment abolished also caspase-3 cleavage in TgCRND8 mice (Figures 3i and j) (Two-way ANOVA, interaction $P<0.05$,
Bonferroni post-hoc test). D-JNKI1 chronic treatment completely blocks biochemical alterations of PSD of TgCRND8.

Specific JNK inhibition protects spines from dysmorphogenesis. To study structural alterations of excitatory synapses we quantitatively analyzed the ultrastructure of volumes of neutrophil sampled at the level of the stratum radiatum of the hippocampus (Figure 4a) of three groups of mice: TgCRND8 mice that had been treated with D-JNKI1 for 5 months (total volume analyzed: $608.82 \mu \mathrm{m}^{3}$ ); age-matched wt $\left(926.43 \mu \mathrm{m}^{3}\right)$ and TgCRND8 $\left(973.41 \mu \mathrm{m}^{3}\right)$ mice that had been injected with the vehicle during the same period of time (Figures 4b-e). We compared the PSD area of the excitatory synapses on spines and found significant differences among the three groups: in the vehicle-treated TgCRND8 mice PSD area was significantly smaller than in the Wt mice $(30 \%$ reduction), whereas the D-JNKI1 treatment reversed this difference of area to the wild-type mice (Figure 4d) (One-way ANOVA, $P<0.05$, Bonferroni post-hoc test). The measurements of the spine volume showed a similar sequence of results (32\% reduction) (Figures $4 \mathrm{c}$ and e) (One-way ANOVA, $P<0.05$, Bonferroni post-hoc test). The EMobservations show a similar trend as the biochemical data and are in line with the hypothesis that D-JNKI1 treatment reverse synaptic dysmorphogenesis in TgCRND8 mice.

Concerning spine loss, we found no differences in total synaptic densities among the three groups of mice: $\mathrm{Wt}$ mice $\left(2.37 \pm 0.30\right.$ synapses per $\left.\mu \mathrm{m}^{3}\right)$; vehicle-treated TgCRND8 mice $(2.53 \pm 0.05)$; and the D-JNKI1-treated mice $(2.64 \pm 0.04)$ (data not shown).

JNK-specific inhibition arrests synaptic impairment in TgCRND8 mice. We previously proved that a chronic administration of D-JNKI1 rescued cognitive impairment and deficits of long-term potentiation (LTP) of excitatory postsynaptic response in the CA1 region of TgCRND8 mice. ${ }^{29}$ We here explored the effect of D-JNKI1 treatment on long-term depression (LTD). LTD is an activity-dependent physiological phenomenon occurring in the dentate gyrus (DG) that also involves synaptic release of glutamate. Previously, it was shown that LTD in the DG was increased in models of $A D{ }^{6}$ We therefore investigated the basis for the functional recovery after D-JNKI1 treatment by measuring LTD in hippocampal slices from TgCRND8 and Wt mice. As shown in Figures 5a-c, a low-frequency stimulation (LFS) protocol applied in slices from 9-months-old $\mathrm{Tg}$ mice produced an abnormally enhanced LTD in the DG as compared to LTD measured in slices from age-matched $\mathrm{Wt}$ animals (reduction of population spike (PS) amplitude, Tg $48 \pm 3.9 \%$ versus $\mathrm{Wt} 19.3 \pm 5.9 \%$; Two-way ANOVA, $P<0.001)$. TAT-control peptide did not have any effects on LTD (data not shown) as already reported for previous studies. ${ }^{29}$ In order to test whether the acute effect of D-JNKI1 in recovering spine function in $\mathrm{Tg}$ mice correlated with a possible recovery to physiological levels of the altered DG LTD, we performed field potential recordings in hippocampal slices treated with D-JNKI1. LFS protocol applied in slices from Tg mice incubated with D-JNKI1 ( $3 \mu \mathrm{M}$ for $2 \mathrm{~h}$ ) produced LTD of synaptic transmission in the DG region that was smaller in respect to LTD measured in non-treated slices 
a

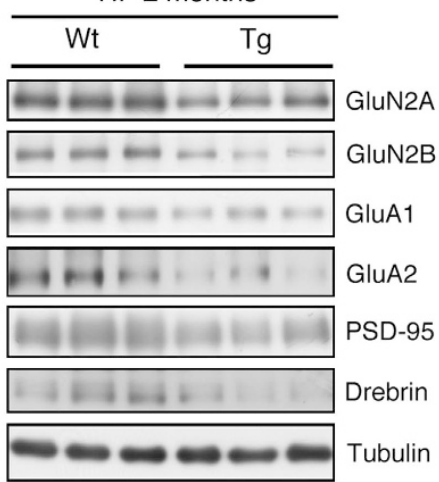

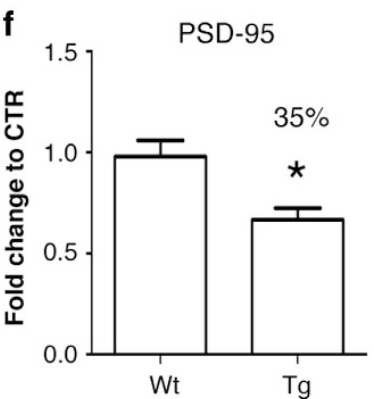

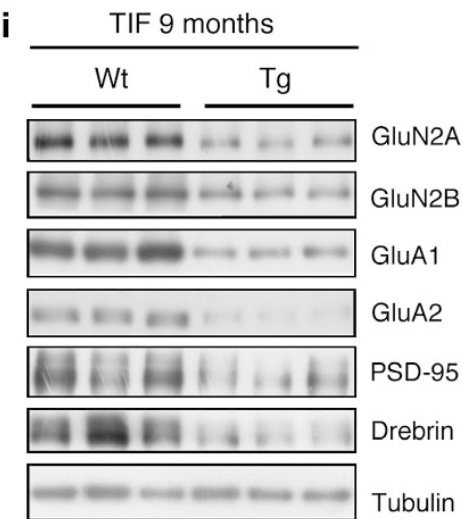

m

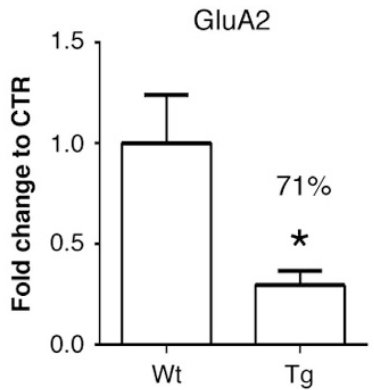

b

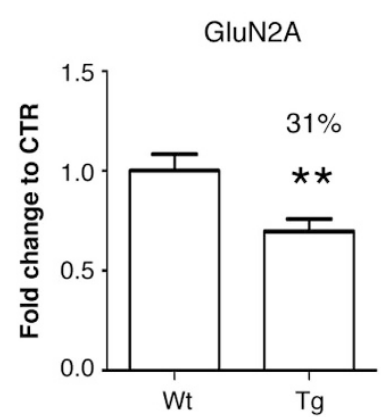

j

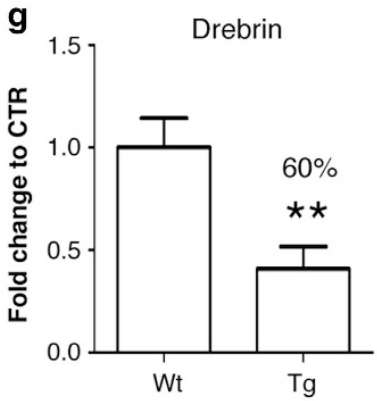

GluN2A
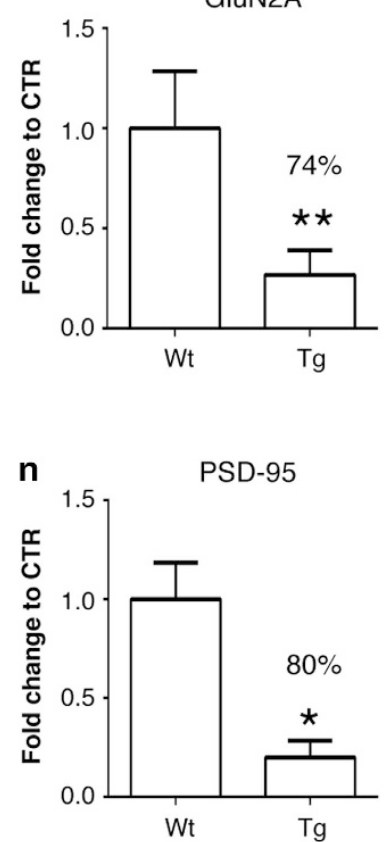

c

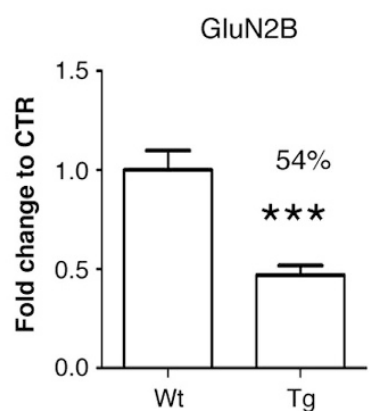

k

d
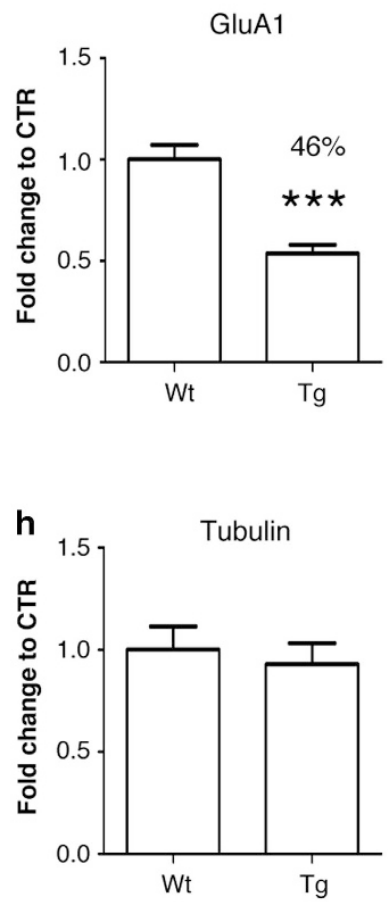

I
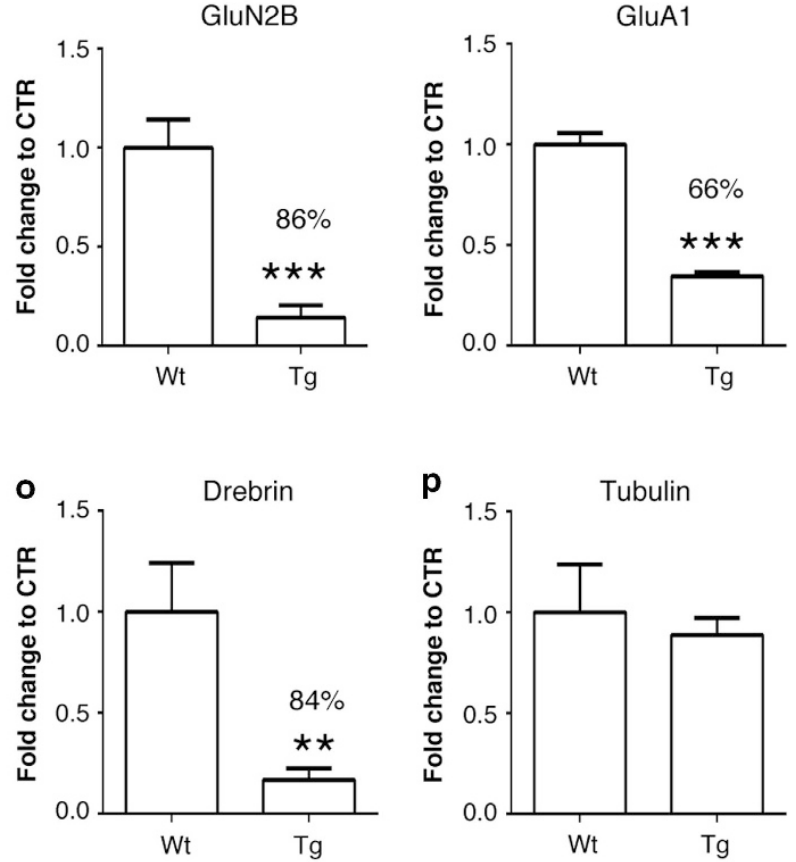

Figure 1 Synaptopathy occurred in TgCRND8 mice, already at an early stage of AD pathology. (a-h) Western blot and relative quantification performed on the TIF fraction of 2-months-old Wt and TgCRND8 mice. Tg mice showed a significant reduction in the PSD levels of GluN2A (31\%) (b) and of GluN2B (54\%) (c) subunits of NMDAr, as well as reduction of GluA1 (46\%) (d) and of GluA2 (35\%) (e) subunits of AMPAr, of PSD-95 (35\%) (f) and of drebrin ( $60 \%$ ) (g) if compared with age-matched Wt mice (Student's $t$-test, ${ }^{*} P<0.05,{ }^{* *} P<0.01,{ }^{* * *} P<0.001, n=6$ ). Tubulin levels were not affected (h) (Student's $t$-test, $P>0.05, n=6$ ). (i-p) Western blot and relative quantification performed on the TIF fraction of 9-months-old Wt and TgCRND8 mice. Tg mice showed a severe reduction in the PSD levels of GluN2A (74\%) (j) and of GluN2B (86\%) (k) subunits of NMDAr, as well as reduction of GluA1 (66\%) (I) and of GluA2 (71\%) (m) subunits of AMPAr, of PSD-95 $(80 \%)$ (n) and of drebrin (84\%) (o) if compared with age-matched Wt mice (Student's $t$-test, ${ }^{*} P<0.05,{ }^{* *} P<0.01,{ }^{* \star *} P<0.001, n=6$ ). Tubulin levels were not affected $(\mathbf{p})$ (Student's $t$-test, $P>0.05, n=6$ ) 
a

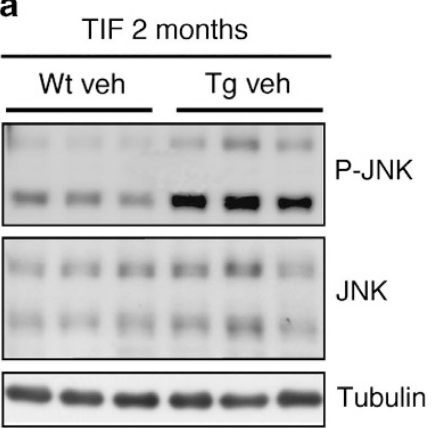

c TIF 9 months

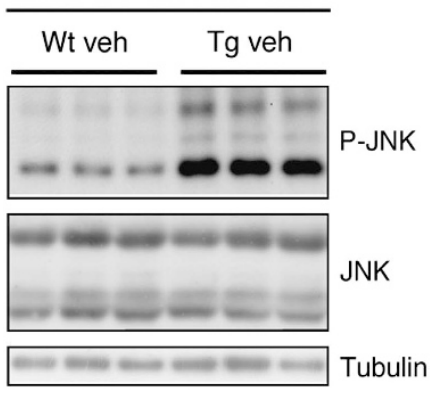

b P-JNK/JNK 2 months

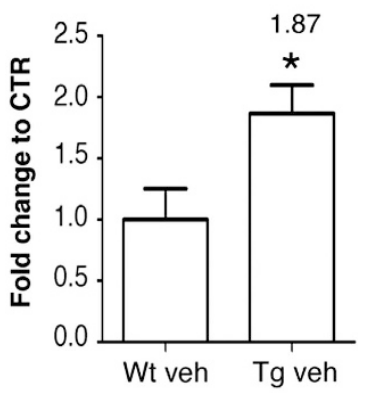

d P-JNK/JNK 9 months

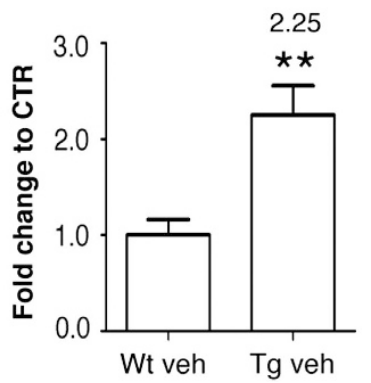

Figure 2 JNK and caspase-3 pathways were activated in the postsynaptic compartment in TgCRND8 mice. ( $\mathbf{a}$ and $\mathbf{b}$ ) Western blot and relative quantification showing P-JNK and JNK levels in the TIF fraction of 2-months-old Wt and TgCRND8 mice. P-JNK/JNK ratio was increased by 1.87 -fold in TgCRND8 mice if compared with the age-matched Wt mice (Student's $t$-test, ${ }^{*} P<0.05, n=6$ ). (c and d) Western blot and relative quantification showing P-JNK and JNK levels in the TIF fraction of 9-months-old Wt and TgCRND8 mice. P-JNK/JNK ratio was increased by 2.25-fold in TgCRND8 mice if compared with the age-matched Wt mice (Student's t-test, ${ }^{*} P<0.01, n=6$ )

from $\mathrm{Tg}$ mice (reduction of PS amplitude, D-JNKI1 $32.1 \pm 3.7 \%$ versus non-treated slices from $\mathrm{Tg}$ mice, Two-way ANOVA, $P<0.001)$. Thus, JNK-specific inhibition with D-JNKI1 significantly reversed the alteration of LTD observed in transgenic mice.

\section{Discussion}

In this study, we unveil a key role of JNK in triggering the intracellular cascade leading to synaptopathy in TgCRND8 mice. JNK activation correlates with the first biochemical PSD changes observed in TgCRND8 mice at 2 months of age, before the onset of cognitive symptoms. These results are in line with our in vitro studies showing that JNK activation anticipates PSD changes in neurons treated with soluble $\mathrm{A} \beta$ oligomers. ${ }^{30}$ Moreover, specific inhibition of JNK completely prevents degeneration of the postsynaptic compartment of excitatory synapses in an in vivo model of $A D$, even if the treatment was commenced after the onset of $A D$ symptoms. This forms a proof that JNK has a pivotal role in synaptopathy and that synaptic degeneration is reversible. Dysfunctional spines have in fact a large plasticity window, as their changes can be reverted also two months after the beginning of the spine degenerative process. The temporal window for pharmacological intervention can thus be extended to a 'post-symptomatic' phase.

Unfortunately, the limitation is that none of the AD animal models available reproduce exactly the human AD pathology, since they do not show neuronal death. However, they are useful tools to study synaptic dysfunction. Among AD animal models, TgCRND8 mice showed a more extensive spectrum of synaptic alterations compared to other AD mouse models, characterized by mutations only in the APP gene (APP23 and Tg2576 mice). In fact, Tg2576 mice were reported to have decreased levels of GluA1, ${ }^{31}$ while APP23 mice showed alterations in the trafficking of synaptic NMDAR subunits GluN2A and GluN2B, in the absence of PSD-95 and AMPAr deficits. ${ }^{32}$ Importantly, TgCRND8 mice develop memory impairment at 3 months of $a^{27}$ and then convincing long-term potentiation (LTP) deficits, ${ }^{29}$ making them a suitable model for pre-clinical pharmacological studies.

To investigate the role of JNK in the induction of synaptopathy and the neuroprotective effect of its inhibition, we administered D-JNKI1 to TgCRND8 mice. ${ }^{27,29}$ We used a 'post-symptomatic' treatment as it is clinically more relevant. This treatment protocol was also supported by our results that demonstrate that JNK activation started at 2 months and persisted with a significant increase during AD progression. D-JNKI1 treatment completely reverted spine alterations: preventing biochemical alterations of the PSD, restoring the levels of NMDAr and AMPAr as well as PSD-95 and drebrin in vivo as it did in vitro. ${ }^{30}$ Moreover, D-JNKI1 abolished at the postsynaptic compartment activation of caspase-3, a pathway implicated in AD spine death. ${ }^{30,31}$

To correlate the biochemical changes of the PSD regions with morphological alterations at synapses, we performed ultrastructural analysis of dendritic spines in the stratum radiatum of the hippocampus. ${ }^{30}$ We demonstrated that D-JNKI1 promotes a structural rescue of excitatory synapses, as the PSD area and the volume of dendritic spines in D-JNKI1-treated TgCNRD8 mice were compared with Wtmice levels. The ultrastructural observations are in line with the biochemical data. In fact, the biochemical analysis revealed that D-JNKI1 completely rescued alterations of the PSD composition. However, we notice an inconsistency between the EM (30\% reduction) and the biochemistry $(70 \%$ reduction) quantification of the PSD region. This quantitative difference between biochemical and ultrastructural data sets could be explained by the fact that ultrastructural 3D reconstructions were performed in a restricted region of the hippocampus whereas the biochemical studies on the PSD region were conducted using the whole hippocampus. Unfortunately, TgCNRD8 did not reveal spine loss in the brain region at the age studied. These results are supported by other studies that described the presence of alterations in spine morphology in the absence of changes in spine numbers in others $A D$ mouse models. ${ }^{33,34}$ Even without evident spine loss, TgCNRD8 mice showed synaptic dysfunction at 9 months of age and presented cognitive deficits assessed with both behavioral and electrophysiological analysis. ${ }^{29}$ Although the protective effect of D-JNKI1 on the density of spines and synapses could not be proven in this model, we have already shown that D-JNKI1 prevented spine loss as well as PSD biochemical alterations ${ }^{30}$ in an in vitro model of synaptopathy triggered by $\mathrm{A} \beta$-soluble toxic 
a

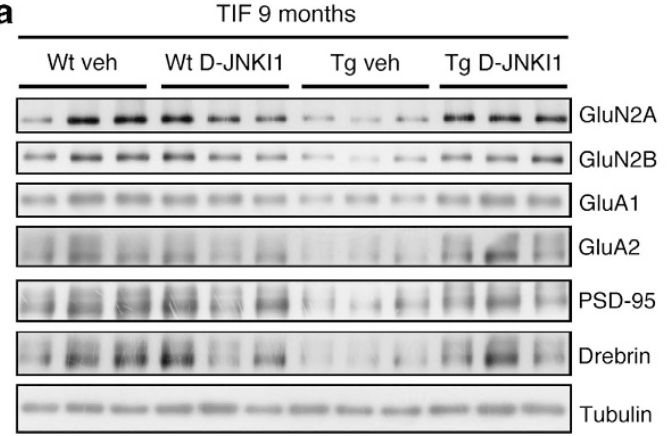

b

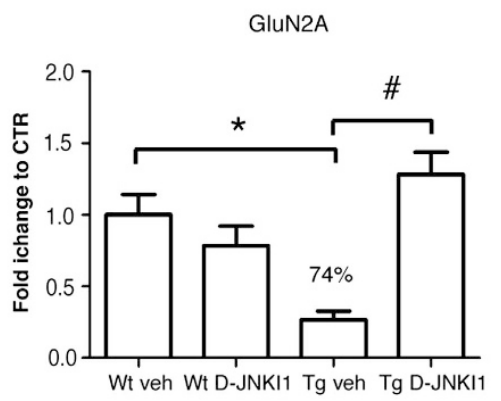

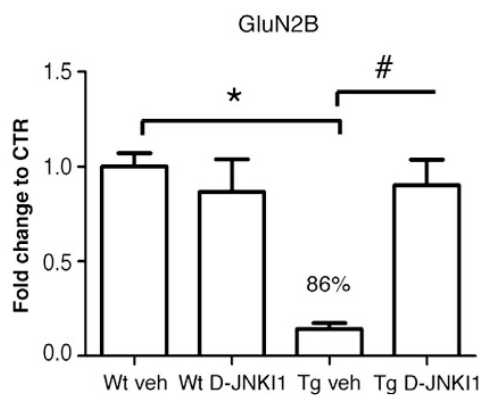

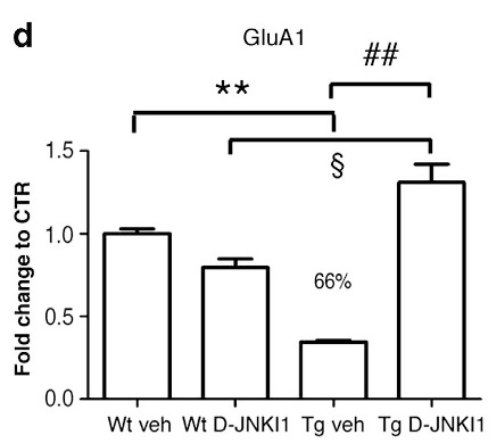

e
GluA2

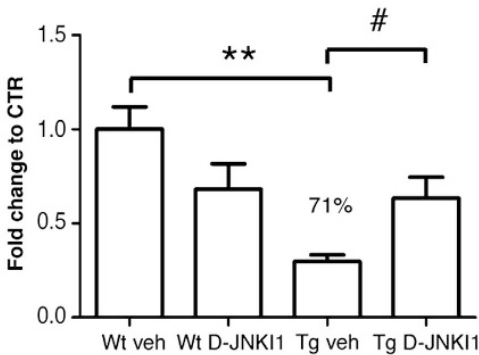

f

g

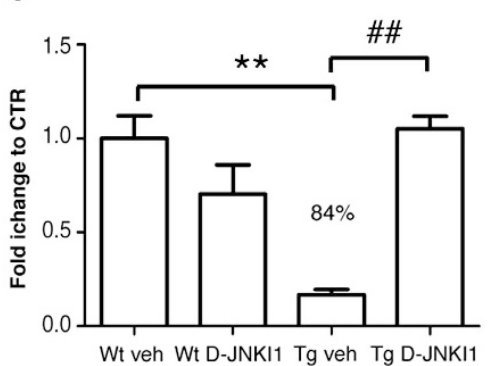

i

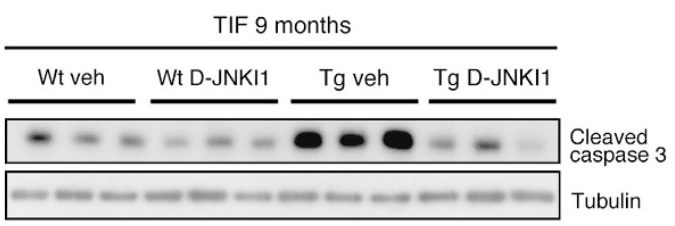

h

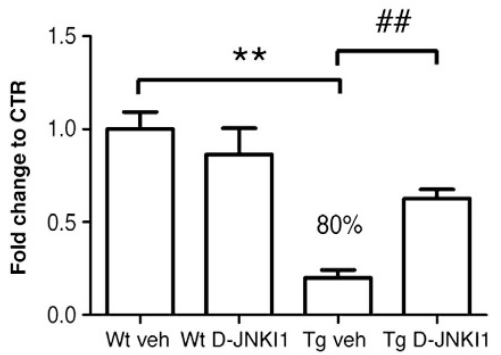

Tubulin

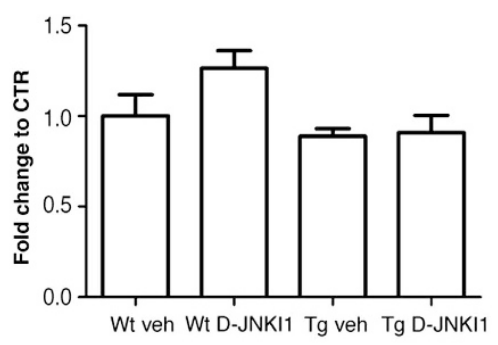

j

Cleaved caspase 3

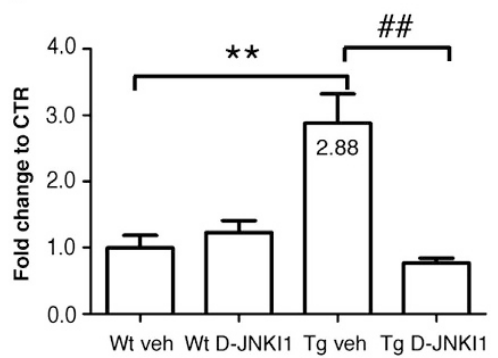

Figure 3 D-JNKI1 prevented A $\beta$-oligomers-induced loss of postsynaptic proteins from the PSD in TgCRND8 mice. (a-h) Western blot and relative quantification of TIF fraction obtained from hippocampus homogenate of 9-months-old Wt and TgCRND8 mice, chronically treated with vehicle (water) or D-JNKI1 (22 mg/kg for 5 months). TgCRND8 mice treated with vehicle showed a strong loss of GluN2A (b) and GluN2B (c) subunits of NMDAr, GluA1 (d) and GluA2 (e) subunits of AMPAr, PSD-95 (f) and drebrin (g) from the PSD. D-JNKI1 chronic treatment completely prevented these alterations in Tg mice, whereas it did not affect protein levels in Wt mice (two-way analysis of variance (ANOVA), Bonferroni post-hoc test, ${ }^{*} P<0.05,{ }^{* \star} P<0.01$ Wt veh versus Tg veh; ${ }^{\#} P<0.05,{ }^{\# \#} P<0.01 \mathrm{Tg}$ veh versus Tg D-JNKI1; ${ }^{\S} P<0.05$ Wt D-JNKl1 versus Tg D-JNKI1, $n=6$ ). Tubulin levels ( $h$ ) were not affected by the treatment or by mice genotype. (i and j) Western blot and relative quantification showing cleaved caspase-3 levels in the TIF fraction of 9-months-old Wt and TgCRND8 mice treated with vehicle (water) or D-JNKI1 (22 mg/kg for 5 months). D-JNKI1 chronic treatment completely prevented activation of caspase-3 in TgCRND8 mice (Two-way ANOVA, Bonferroni post-hoc test, ${ }^{\star *} P<0.01 \mathrm{Wt}$ veh versus $\mathrm{Tg}$ veh; ${ }^{\text {\#\#} ~} P<0.01 \mathrm{Tg}$ veh versus $\mathrm{Tg}$ D-JNKI1, $n=6$ )

oligomers. A longitudinal study on synaptic density changes in the hippocampus of TgCNRD8 mice would be needed to identify the onset of the loss of excitatory synapses in this mouse model of AD.
LTD recordings were performed to correlate the biochemical/morphological changes to the altered synaptic functionality in TgCNRD8 mice. LTD is thought to result mainly from a decrease in the PSD size and composition and strongly 
a

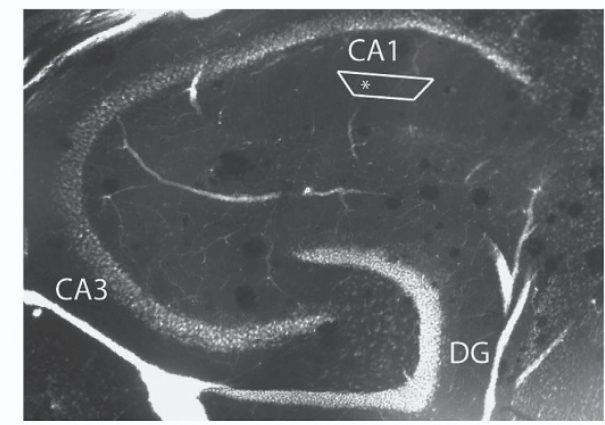

b

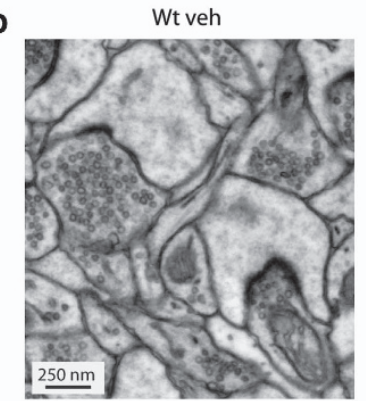

Wt veh
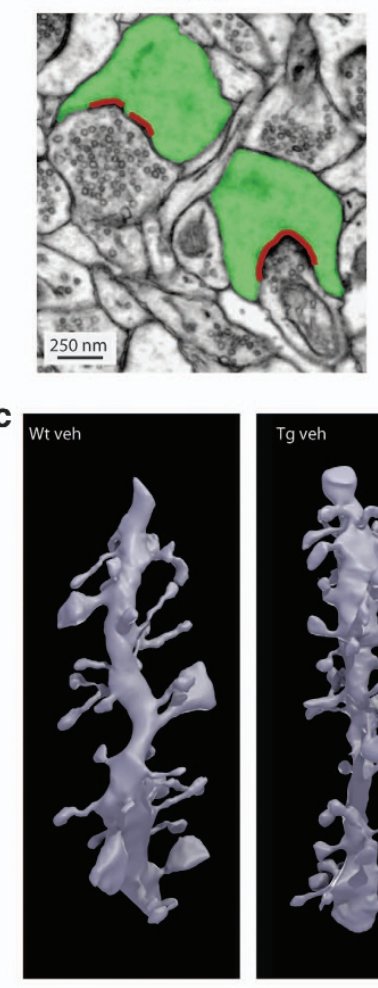

Tg veh

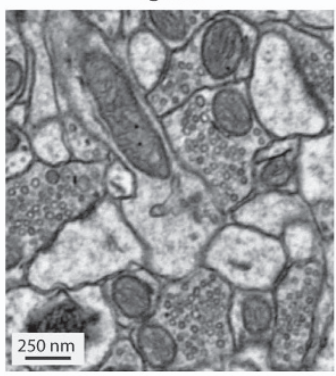

$\mathrm{Tg}$ veh
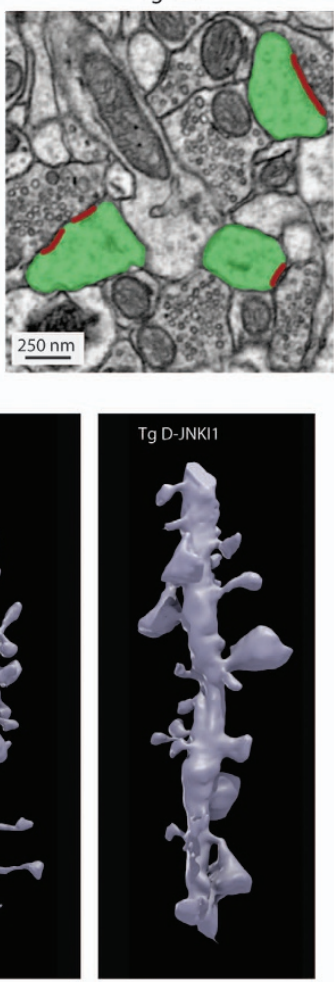

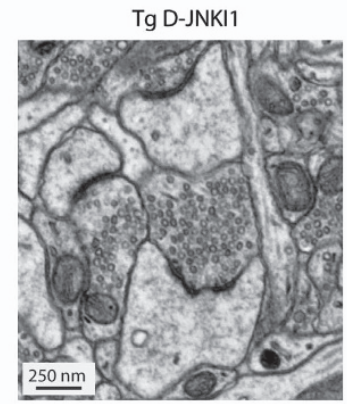

Tg D-JNKI1

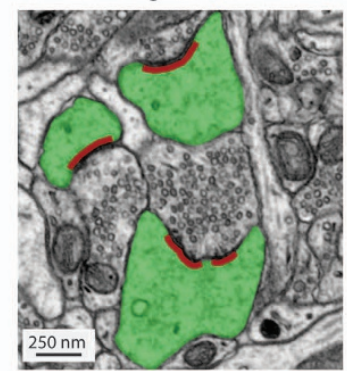

d

PSD AREA

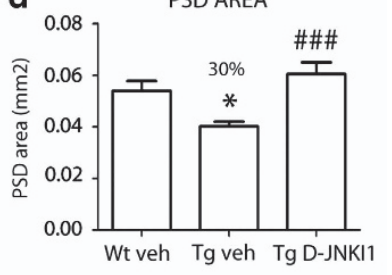

e

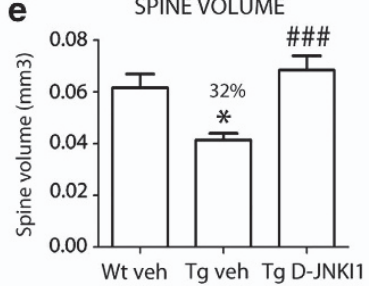

Figure 4 D-JNKI1 prevented morphological alterations in dendritic spines observed in TgCRND8 mice. Alteration in spine morphology was assessed by performing serial sectioning electron microscope analysis. (a) Micrograph of a section through the hippocampus showing the position of the neutrophil sampled at the level of the stratum radiatum of the hippocampus. The black box shows the position of the block whereas the asterisk represent the site of the analysis. (b) Electron micrographs showing excitatory synapses on spines in TgCRND8 mice treated with vehicle (Tg veh) or with $22 \mathrm{mg} / \mathrm{kg}$ D-JNKI1 (Tg D-JNKI1). D-JNKI1 treatment induced an increase in the PSD (red in figure) area and in the postsynaptic spine (green in figure) volume if compared with vehicle-treated TgCRND8 mice. (c) Three-dimensional EM reconstruction illustrating dendritic segments from TgCRND8 mice treated with vehicle or D-JNKI1; note the appearance of large spines in the D-JNKI1-treated case. (d and e) Graphs showing the effect of D-JNKI1 treatment on PSD area (d) and in spine volume (e) in TgCRND8 mice. TgCRND8 mice show a significant reduction in the PSD area (30\%) (d) and spine volume $(32 \%)\left(\right.$ e). D-JNKI1 treatment restores normal sizes of spines and PSD area (one-way ANOVA, Bonferroni post-hoc test, ${ }^{*} P<0.05 \mathrm{Wt}$ veh versus Tg veh; ${ }^{\# \# ~} P<0.001$ Tg veh versus Tg D-JNKI1, Wt veh $n=122$ spines, Tg veh $n=168$ spines; TgCRND8 D-JNKI1 $n=178$ spines) 
a
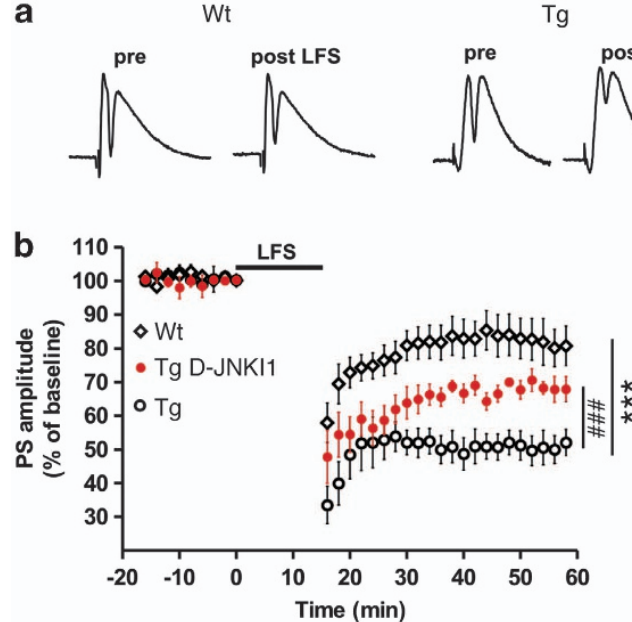

$\operatorname{Tg}$

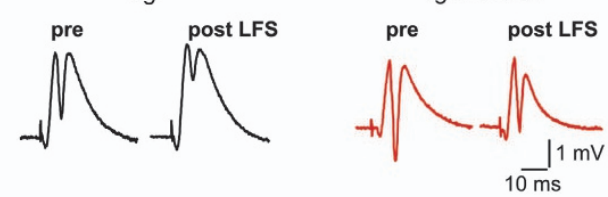

C

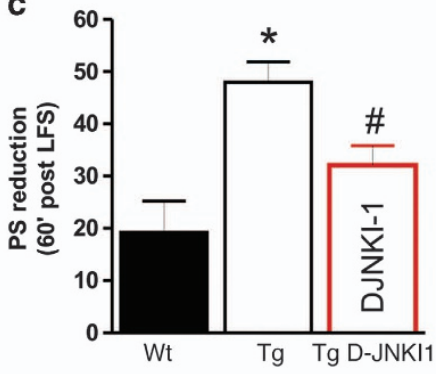

Figure 5 D-JNKI1 restored synaptic function in TgCRND8 mice. Electrophysiological analysis. (a) Example pairs of traces of field potentials showing population spikes (PS) evoked before and after the induction of LTD by a LFS protocol in hippocampal slices cut from Wt (left) and TgCRND8 mice (center) and in hippocampal slices from Tg mice incubated with D-JNKI1 ( $3 \mu \mathrm{M}, 2 \mathrm{~h})$ (right). (b) Time-course graph of population spike (PS) amplitudes showing LTD induced by LFS protocol in slices from Wt mice (diamonds), Tg mice (open circles) and in slices from Tg mice incubated with D-JNKI1 (red-filled circles) (two-way ANOVA, ${ }^{* \star *} P<0.001 \mathrm{Wt}$ versus Tg, \#\#\# $P<0.001 \mathrm{Tg}$ versus Tg D-JNKI1; Wt $n=11, \operatorname{Tg} n=8, \operatorname{Tg}$ D-JNKI1 $n=4$ ). (c) Histogram summarizing the reduction of the PS amplitude measured in control slices from Tg (white bar) compared with Wt mice (black bar) (Student's $t$-test, ${ }^{*} P<0.05$ ), and in slices from Tg mice incubated with D-JNKI1 (red bar) compared with the non-treated control slices from Tg mice (Student's t-test, ${ }^{\#} P<0.05$ )

correlates with synaptopathy. ${ }^{35,36}$ TgCRND8 mice presented an increased LTD, which was reverted by D-JNKI1 treatment in 9-months-old AD animals. Thus, JNK inhibition normalized the altered hippocampal synaptic plasticity by restoring LTD impairment as well.

All together, these data indicate that JNK activation triggers and controls synaptopathy in TgCNRD8 transgene-dependent phenotype mice. Moreover, JNK inhibition prevents biochemical, morphological and functional alterations found in TgCRND8 spines, even if the treatment is started after the onset of AD pathology.

Until now, few studies addressed protection against synaptopathy in vivo. Among them, there are controversial data regarding the modulation of the $\gamma$-secretase. The selective blocker CHF5074 of this enzyme restored synaptic plasticity in Tg2576 mice, ${ }^{37}$ although it did not rescue synaptic defects in a mouse model of familial Danish dementia. ${ }^{38}$ In addition, Chen et al. ${ }^{39}$ showed that an inhibitor of calcineurin completely prevented $\mathrm{A} \beta$-induced LTP deficits in wt mice. However, further comparison with our observations is difficult as these studies did not report any data regarding biochemical and morphological changes at synapses.

A recent report indicated that the pharmacological inhibition of caspase-3 rescued synaptic failure and memory deficits in Tg2576 mice. ${ }^{31}$ In this study, the authors proved that inhibition of caspase- 3 reduced GluA1 dephosphorylation and removal from the $\mathrm{PSD}$, and also reversed hippocampal function deficits at the onset of AD.

D-JNKI1 neuroprotective effect succeeded in preventing not only GluA1 loss at the PSD of glutamatergic synapses but also GluA2 subunits of AMPAr, GluN2A and GluN2B subunits of NMDAr, as well as two important postsynaptic proteins: PSD-95 and drebrin. This probably suggests that JNK controls the removal of both AMPAr and NMDAr by regulating PSD-95 abundance at the PSD as suggested in another model. ${ }^{40}$ PSD-95 and caspase-3 have been identified as JNK targets. ${ }^{40,41}$ In fact, D-JNKI1 blocked both PSD-95 loss and caspase-3 cleavage in the PSD region, preventing spine degeneration. ${ }^{30}$ Inhibition of JNK is therefore the most efficient neuroprotective strategy currently known against synaptopathy.

D-JNKI1 treatment was conducted 1 month after the onset of cognitive defects and PSD changes in TgCRND8 mice. The JNK inhibition completely prevented glutamatergic synaptopathy, indicating a high potentiality of the treatment within an interesting window of intervention. We believe that the powerful effect obtained with D-JNKI1 is linked to its outcome on maintenance of the postsynaptic element structure and functionality, but it is also due to its effect on APP phosphorylation and cleavage, which contributes to $A \beta$ oligomers formation, LTP impairment ${ }^{29}$ and Tau hyperphosphorylation. ${ }^{42}$ In fact, we had previously proven that JNK is involved in APP phosphorylation at Thr668 and is consequently implicated in the production of soluble $\mathrm{A} \beta$ oligomers. Taken together these results suggest that JNK is a key pathway in AD; in fact, JNK triggers spine dysfunction but it also contributes to the progression of the pathology regulating the production of the toxic soluble $\mathrm{A} \beta$ oligomers.

D-JNKI1 was designed to prevent C-Jun phosphorylation, ${ }^{43,44}$ which regulates a multitude of intracellular signaling related to stress and cell death. ${ }^{45}$ This is another important effect carried in TgCRND8 mice that explains its powerful neuroprotective role in vivo.

In conclusion, all together these results set the basis for the development of a JNK-based therapeutic strategy targeting synapses for the treatment of AD.

\section{Materials and Methods}

Transgenic mice and pharmacological treatments. Experimental procedures on animals were conducted in accordance with the European Communities Council Directive (86/609/EEC) and were authorized by Italian legal 
guidelines. All efforts were made to minimize the number of animals used and their suffering. TgCRND8 mice ${ }^{27}$ were housed at $23^{\circ} \mathrm{C}$ room temperature with food and water ad libitum and a 12-h-light/dark cycle. TgCRND8 mice of either sex were treated chronically with D-JNKI1 (IRCCS-Istituto di Ricerche Farmacologiche 'Mario Negri', Milan, Italy) diluted in water $(22 \mathrm{mg} / \mathrm{kg})$ or with water as vehicle starting at 4-5 months of age. Mice received an intraperitoneal injection every 21 days for 5 months (six injections).

Subcellular fractionation (TIF). Subcellular fractionation was performed as reported in the literature with minor modifications. ${ }^{28}$ Briefly, hippocampi were dissected and homogenized in $0.32 \mathrm{M}$ ice-cold sucrose buffer containing the following (in mM): $1 \mathrm{HEPES}, 1 \mathrm{MgCl}_{2}, 1 \mathrm{EDTA}, 1 \mathrm{NaHCO}_{3}$ and $0.1 \mathrm{PMSF}$, at pH 7.4, in the presence of a complete set of protease inhibitors (Complete; Roche Diagnostics, Basel, Switzerland) and phosphatases inhibitors (Sigma, St. Louis, MO, USA). Samples were centrifuged at $1000 \times g$ for $10 \mathrm{~min}$. The resulting supernatant (S1) was centrifuged at $13000 \times g$ for 15 min to obtain a crude membrane fraction (P2 fraction). The pellet was resuspended in $1 \mathrm{mM}$ HEPES plus protease and phosphatise inhibitor in a glass-glass potter and centrifuged at $100000 \times g$ for $1 \mathrm{~h}$. The pellet (P3) was resuspended in buffer containing $75 \mathrm{mM}$ $\mathrm{KCl}$ and $1 \%$ Triton $\mathrm{X}-100$ and centrifuged at $100000 \times g$ for $1 \mathrm{~h}$. The final pellet (P4) referred to as TIF was homogenized in a glass-glass potter in $20 \mathrm{mM}$ HEPES and stored at $-80^{\circ} \mathrm{C}$ until processing.

Western blot. Protein concentrations were quantified using the Bradford Assay (Bio-Rad Protein Assay 500-0006, Munchen, Germany), and $10 \mu \mathrm{g}$ of whole-cell proteins or brain homogenates and $5 \mu \mathrm{g}$ of TIF-extracted proteins were separated by $10 \%$ SDS polyacrylamide gel electrophoresis. PVDF membranes were blocked in Tris-buffered saline ( $5 \%$ no-fat milk powder, $0.1 \%$ Tween 20$)(1 \mathrm{~h}$, room temperature). Primary antibodies were diluted in the same buffer (incubation overnight, $\left.4^{\circ} \mathrm{C}\right)$ using: anti GluN2A (1:2000, Gibco-Invitrogen, Paisley, Scotland, UK), anti GluN2B (1:2000, Gibco-Invitrogen), anti GluA1 (1:1000, Millipore, Billerica, MA, USA), anti GluA2 (1:1000, Millipore), anti PSD-95 (1:2000, Cayman Chemical Company, Ann Arbor, MI, USA), anti drebrin (1:2000, Assay Design, Ann Arbor, MI, USA), P-JNK (G-7) (1:1000, Cell Signaling Technology, Danvers, MA, USA), JNK (1:1000, Cell Signaling Technology), Cleaved caspase3 (1:1000, Cell Signaling Technology). All blots were normalized to Tubulin (1:5000, Santa Cruz Biotechnology, Santa Cruz, CA, USA) and at least three independent experiments were performed. Blots were developed using horseradish peroxidase-conjugated secondary antibodies and the ECL chemiluminescence system. Western blots were quantified by densitometry using Quantity One software (Bio-Rad, Hercules, CA, USA).

Serial section electron microscopy. Vehicle- and D-JNKI1-treated TgCRND8 mice and age-matched wild-type mice (age: 9 months; number of mice used: total of eight; Wt veh $n=2$, Tg veh $n=3$ and Tg D-JNKI1 $n=3$ ) were deeply anesthetized with Nembutal $(60 \mathrm{mg} / \mathrm{kg}$, i.p.) and perfused through the heart with $300 \mathrm{ml}$ of fixative $(2.5 \%$ glutaraldehyde and $2 \%$ paraformaldehyde in cacodylate buffer, $0.1 \mathrm{M}(\mathrm{pH}$ 7.4). One hour after perfusion, the brain was removed and vibratome sections were cut at $60 \mu \mathrm{m}$ tangential to the dorsal surface of the brain. Sections were washed in $0.1 \mathrm{M}$ cacodylate buffer, stained with $1.5 \%$ potassium ferrocyanide in osmium tetroxide ( $1 \%$ in $0.1 \mathrm{M}$ cacodylate buffer, $\mathrm{pH} 7.4$ ), and with osmium tetroxide alone, then with uranyl acetate. Sections were dehydrated in alcohol and in propylene oxide and embedded in Durcapan ACM resin (Fluka, Neu-Ulm, Germany) between silicon-coated glass slides.

After the resin hardened, the stratum radiatum of $\mathrm{CA} 1$ region of the hippocampus was identified and trapezoid blocks were prepared within this region. Series of 100-130 sections were cut at $50 \mathrm{~nm}$ (ultracut UCT microtome; Leica, Wetzlar, Germany) and collected on Formvar support film on single-slot copper grids. Sections were washed in bi-distilled water and contrasted with lead citrate.

Serial images of the stratum radiatum part of CA1 region were collected using Morada CCD camera and iTEM software (Olympus, Munster, Germany) on a Philips CM10 electron microscope (filament voltage $80 \mathrm{kV}$ ), aligned and analyzed using Fiji software (http://fiji.sc/wiki/index.php/Fiji).

Morphometric analysis of spines and synapses. Morphometric analysis on the densities of excitatory and inhibitory synapses on shaft and spines was accomplished on volumes of neuropil from Wt veh $(n=2$; total volume analyzed: $\left.609 \mu \mathrm{m}^{3}\right)$, Tg veh $\left(n=3\right.$; total volume analyzed: $\left.973 \mu \mathrm{m}^{3}\right)$ and $\mathrm{TgD}$ JNKI1 $\left(n=3\right.$; total volume analyzed: $\left.926 \mu \mathrm{m}^{3}\right)$ mice.
The thickness of the sections in the stack was calculated by measuring the diameter of longitudinally cut mitochondria and determining the number of sections in which the mitochondria were found as previously described. ${ }^{46}$

Synapses were identified by vesicle number $(n>3)$, shape, clarity and postsynaptic density (PSD) thickness across electron micrographs taken from three consecutive sections. Spine volumes and PSD area were measured on randomly selected asymmetric synapses (Wt veh, $n=122$, Tg veh, $n=168 ; \mathrm{Tg}$ D-JNKI1, $n=179$ ) within the analyzed volume of each mouse. For PSD-area analysis, a line segment was drawn along the postsynaptic density (the electron dense plaque on the postsynaptic membrane opposed to the presynaptic element) in each section through a given synapse using polyline tool in Fiji software. For each synapse, the sum of the length of the lines was multiplied by the section thickness resulting in the PSD area. The analysis was made in double-blind, that is, without knowing the identity of the animal under study.

Electrophysiology. Mice were decapitated and the brain was removed and immersed for 2-3 min in ice-cold artificial CSF (ACSF) containing (in $\mathrm{mM}$ ): 126 $\mathrm{NaCl}, 2.5 \mathrm{KCl}, 1.2 \mathrm{MgCl}_{2}, 1.2 \mathrm{NaH}_{2} \mathrm{PO}_{4}, 2.4 \mathrm{CaCl}_{2}, 10$ glucose and $25 \mathrm{NaHCO}_{3}$, continuously bubbled with $95 \% \mathrm{O}_{2}$ and $5 \% \mathrm{CO}_{2}, \mathrm{pH} 7.4$. The hippocampus was extracted and cut in ice-cold ACSF with a vibratome (Pelco 1000 plus; Redding, CA, USA) into 400- $\mu \mathrm{m}$-thick transverse slices, which were allowed to recover in oxygenated ACSF at $30^{\circ} \mathrm{C}$ for $30 \mathrm{~min}$, and then at room temperature for another $1-2 \mathrm{~h}$ before experimental recordings. For D-JNKI1 treatment, slices were incubated in oxygenated ACSF containing $3 \mu \mathrm{M}$ D-JNKI1 for $2 \mathrm{~h}$ before the onset of recording. A slice was transferred into the recording chamber and submerged in ACSF at a constant rate of $2.5 \mathrm{ml} / \mathrm{min}$ at $30^{\circ} \mathrm{C}$. Recording electrodes were made of borosilicate glass capillaries (GC150F-10; Harvard Apparatus, Holliston, MA, USA) and filled with $2 \mathrm{M} \mathrm{NaCl}$ (resistance 10-15 M 2 ). Under visual control, the stimulating electrode was inserted into the Perforant pathway, and the recording electrode into the granular layer of the dentate gyrus (DG). Testing stimuli at $0.1 \mathrm{~Hz}, 10 \mu \mathrm{s}$ duration and 20-30 V amplitude evoked field potential responses of $50-70 \%$ of maximal amplitude, that included population spikes (PS). Field potentials were filtered at $3 \mathrm{KHz}$, digitized at $10 \mathrm{KHz}$ and stored on a PC. An Axoclamp 2B amplifier (Axon Instruments, Union City, CA, USA) was used for extracellular recordings. After recording a stable baseline for $20 \mathrm{~min}$, long-term depression (LTD) was induced by a low-frequency stimulation (LFS) delivered for $15 \mathrm{~min}$ at $1 \mathrm{~Hz}$ at a stimulus used during baseline recording. After LFS onset, the PS amplitude time-course was followed for at least $60 \mathrm{~min}$.

\section{Conflict of Interest}

The authors declare no conflict of interest.

Acknowledgements. This study was supported by San Paolo 2008-2437, Banca Intesa San Paolo grant 2009-2011 to MS, Marie Curie Industry-Academia Partnerships and Pathways (IAPP) CPADS (Cell permeable peptides as drug delivery system) and CARIPLO (Cassa di risparmio delle provincie Lombarde) Foundation 2009-2425, ADDF (Alzheimer's Drugs Discovery Foundation) US grant, Swiss National Science Foundation grant: 31003A_125379. We thank Dr. Gardoni and Prof. Di Luca for their advices and criticisms.

1. Scheff SW, Price DA, Schmitt FA, Mufson EJ. Hippocampal synaptic loss in early Alzheimer's disease and mild cognitive impairment. Neurobiol Aging 2006; 27: 1372-1384.

2. Crews L, Masliah E. Molecular mechanisms of neurodegeneration in Alzheimer's disease. Hum Mol Genet 2010; 19: R12-R20.

3. Gomez-Isla T, Price JL, McKeel Jr DW, Morris JC, Growdon JH, Hyman BT. Profound loss of layer II entorhinal cortex neurons occurs in very mild Alzheimer's disease. J Neurosci 1996; 16: 4491-4500.

4. Lambert MP, Barlow AK, Chromy BA, Edwards C, Freed R, Liosatos M et al. Diffusible, nonfibrillar ligands derived from Abeta1-42 are potent central nervous system neurotoxins. Proc Natl Acad Sci USA 1998; 95: 6448-6453.

5. Lesne S, Koh MT, Kotilinek L, Kayed R, Glabe CG, Yang A et al. A specific amyloid-beta protein assembly in the brain impairs memory. Nature 2006; 440: 352-357.

6. Shankar GM, Li S, Mehta TH, Garcia-Munoz A, Shepardson NE, Smith I et al. Amyloidbeta protein dimers isolated directly from Alzheimer's brains impair synaptic plasticity and memory. Nat Med 2008; 14: 837-842.

7. Ono K, Condron MM, Teplow DB. Structure-neurotoxicity relationships of amyloid betaprotein oligomers. Proc Natl Acad Sci USA 2009; 106: 14745-14750. 
8. Fitzjohn SM, Morton RA, Kuenzi F, Rosahl TW, Shearman M, Lewis $\mathrm{H}$ et al. Age-related impairment of synaptic transmission but normal long-term potentiation in transgenic mice that overexpress the human APP695SWE mutant form of amyloid precursor protein. J Neurosci 2001; 21: 4691-4698.

9. Larson J, Lynch G, Games D, Seubert P. Alterations in synaptic transmission and long-term potentiation in hippocampal slices from young and aged PDAPP mice. Brain Res 1999; 840: 23-35

10. Jung JH, An K, Kwon OB, Kim HS, Kim JH. Pathway-specific alteration of synaptic plasticity in Tg2576 mice. Mol Cells 2011; 32: 197-201.

11. Oddo S, Caccamo A, Shepherd JD, Murphy MP, Golde TE, Kayed R et al Triple-transgenic model of Alzheimer's disease with plaques and tangles: intracellular Abeta and synaptic dysfunction. Neuron 2003; 39: 409-421.

12. Kimura R, Ohno M. Impairments in remote memory stabilization precede hippocampal synaptic and cognitive failures in 5XFAD Alzheimer mouse model. Neurobiol Dis 2009; 33 : 229-235.

13. Trinchese F, Fa M, Liu S, Zhang H, Hidalgo A, Schmidt SD et al. Inhibition of calpains improves memory and synaptic transmission in a mouse model of Alzheimer disease. $J$ Clin Investig 2008; 118: 2796-2807.

14. Gasparini L, Dityatev A. Beta-amyloid and glutamate receptors. Exp Neurol 2008; 212: 1-4.

15. Parameshwaran K, Dhanasekaran M, Suppiramaniam V. Amyloid beta peptides and glutamatergic synaptic dysregulation. Exp Neurol 2008; 210: 7-13.

16. Sze $\mathrm{C}, \mathrm{Bi} \mathrm{H}$, Kleinschmidt-DeMasters BK, Filley CM, Martin LJ. N-Methyl-D-aspartate receptor subunit proteins and their phosphorylation status are altered selectively in Alzheimer's disease. J Neurological Sci 2001; 182: 151-159.

17. Lacor PN, Buniel MC, Chang L, Fernandez SJ, Gong Y, Viola KL et al. Synaptic targeting by Alzheimer's-related amyloid beta oligomers. J Neurosci 2004; 24: 10191-10200.

18. Mishizen-Eberz AJ, Rissman RA, Carter TL, Ikonomovic MD, Wolfe BB, Armstrong DM Biochemical and molecular studies of NMDA receptor subunits NR1/2A/2B in hippocampal subregions throughout progression of Alzheimer's disease pathology. Neurobiol Dis 2004; 15: 80-92.

19. Snyder EM, Nong Y, Almeida CG, Paul S, Moran T, Choi EY et al. Regulation of NMDA receptor trafficking by amyloid-beta. Nat Neurosci 2005; 8: 1051-1058.

20. Shankar GM, Bloodgood BL, Townsend M, Walsh DM, Selkoe DJ, Sabatini BL. Natura oligomers of the Alzheimer amyloid-beta protein induce reversible synapse loss by modulating an NMDA-type glutamate receptor-dependent signaling pathway. J Neurosci 2007; 27: 2866-2875.

21. Almeida CG, Tampellini D, Takahashi RH, Greengard P, Lin MT, Snyder EM et al Beta-amyloid accumulation in APP mutant neurons reduces PSD-95 and GluR1 in synapses. Neurobiol Disease 2005; 20: 187-198.

22. Hsieh H, Boehm J, Sato C, Iwatsubo T, Tomita T, Sisodia S et al. AMPAR removal underlies Abeta-induced synaptic depression and dendritic spine loss. Neuron 2006; 52 831-843

23. Cerpa W, Farias GG, Godoy JA, Fuenzalida M, Bonansco C, Inestrosa NC. Wnt-5a occludes Abeta oligomer-induced depression of glutamatergic transmission in hippocampal neurons. Mol Neurodegenerat 2010; 5: 3

24. Walsh DM, Hartley DM, Kusumoto Y, Fezoui Y, Condron MM, Lomakin A et al. Amyloid beta-protein fibrillogenesis. Structure and biological activity of protofibrillar intermediates. JBiol Chem 1999; 274: 25945-25952.

25. Li S, Jin M, Koeglsperger T, Shepardson NE, Shankar GM, Selkoe DJ. Soluble Abeta oligomers inhibit long-term potentiation through a mechanism involving excessive activation of extrasynaptic NR2B-containing NMDA receptors. J Neurosci 2011; 31: $6627-6638$.

26. Palop JJ, Chin J, Roberson ED, Wang J, Thwin MT, Bien-Ly N et al. Aberrant excitatory neuronal activity and compensatory remodeling of inhibitory hippocampal circuits in mouse models of Alzheimer's disease. Neuron 2007; 55: 697-711.

27. Chishti MA, Yang DS, Janus C, Phinney AL, Horne P, Pearson J et al. Early-onset amyloid deposition and cognitive deficits in transgenic mice expressing a double mutant form of amyloid precursor protein 695. J Biol Chem 2001; 276: 21562-21570.

28. Gardoni F, Schrama LH, Kamal A, Gispen WH, Cattabeni F, Di Luca M. Hippocampa synaptic plasticity involves competition between $\mathrm{Ca}^{2+} /$ calmodulin-dependent protein kinase II and postsynaptic density 95 for binding to the NR2A subunit of the NMDA receptor. J Neurosci 2001; 21: 1501-1509.

29. Sclip A, Antoniou X, Colombo A, Camici GG, Pozzi L, Cardinetti D et al. c-Jun N-terminal kinase regulates soluble Abeta oligomers and cognitive impairment in $A D$ mouse model. J Biol Chem 2011; 286: 43871-43880.

30. Sclip A, Arnaboldi A, Colombo I, Veglianese P, Colombo L, Messa M et al. Soluble Abeta oligomers-induced synaptopathy: c-Jun N-terminal kinase's role. J Mol Cell Biol 2013; 5: 277-279.

31. D'Amelio M, Cavallucci V, Middei S, Marchetti C, Pacioni S, Ferri A et al. Caspase-3 triggers early synaptic dysfunction in a mouse model of Alzheimer's disease. Nat Neurosci 2011; 14: 69-76.

32. Balducci C, Tonini R, Zianni E, Nazzaro C, Fiordaliso F, Salio M et al. Cognitive deficits associated with alteration of synaptic metaplasticity precede plaque deposition in AbetaPP23 transgenic mice. J Alzheimer's Dis 2010; 21: 1367-1381.

33. Merino-Serrais $\mathrm{P}$, Knafo S, Alonso-Nanclares L, Fernaud-Espinosa I, DeFelipe J. Layer-specific alterations to CA1 dendritic spines in a mouse model of Alzheimer's disease. Hippocampus 2011; 21: 1037-1044.

34. Malm TM, livonen H, Goldsteins G, Keksa-Goldsteine V, Ahtoniemi T, Kanninen K et al. Pyrrolidine dithiocarbamate activates Akt and improves spatial learning in APP/PS1 mice without affecting beta-amyloid burden. J Neurosci 2007; 27: 3712-3721.

35. Bastrikova N, Gardner GA, Reece JM, Jeromin A, Dudek SM. Synapse elimination accompanies functional plasticity in hippocampal neurons. Proc Natl Acad Sci USA 2008; 105: 3123-3127.

36. Becker N, Wierenga CJ, Fonseca R, Bonhoeffer T, Nagerl UV. LTD induction causes morphological changes of presynaptic boutons and reduces their contacts with spines. Neuron 2008; 60: 590-597.

37. Balducci C, Mehdawy B, Mare L, Giuliani A, Lorenzini L, Sivilia S et al. The gammasecretase modulator CHF5074 restores memory and hippocampal synaptic plasticity in plaque-free Tg2576 mice. J Alzheimer's Dis 2011; 24: 799-816.

38. Tamayev R, D'Adamio L. Inhibition of gamma-secretase worsens memory deficits in a genetically congruous mouse model of Danish dementia. Mol Neurodegenerat 2012; 7: 19.

39. Chen QS, Wei WZ, Shimahara T, Xie CW. Alzheimer amyloid beta-peptide inhibits the late phase of long-term potentiation through calcineurin-dependent mechanisms in the hippocampal dentate gyrus. Neurobiol Learn Mem 2002; 77: 354-371.

40. Kim MJ, Futai K, Jo J, Hayashi Y, Cho K, Sheng M. Synaptic accumulation of PSD-95 and synaptic function regulated by phosphorylation of serine-295 of PSD-95. Neuron 2007; 56 : 488-502.

41. Repici M, Centeno C, Tomasi S, Forloni G, Bonny C, Vercelli A et al. Time-course of C-Jun $\mathrm{N}$-terminal kinase activation after cerebral ischemia and effect of D-JNKI1 on c-Jun and caspase-3 activation. Neuroscience 2007; 150: 40-49.

42. Ploia C, Antoniou X, Sclip A, Grande V, Cardinetti D, Colombo A et al. JNK plays a key role in tau hyperphosphorylation in Alzheimer's disease models. J Alzheimer's Dis 2011; 26: 315-329.

43. Borsello T, Clarke PG, Hirt L, Vercelli A, Repici M, Schorderet DF et al. A peptide inhibitor of c-Jun $\mathrm{N}$-terminal kinase protects against excitotoxicity and cerebral ischemia. Nat Med 2003; 9: 1180-1186.

44. Bonny C, Oberson A, Negri S, Sauser C, Schorderet DF. Cell-permeable peptide inhibitors of JNK: novel blockers of beta-cell death. Diabetes 2001; 50: 77-82.

45. Weston CR, Davis RJ. The JNK signal transduction pathway. Curr Opin Cell Biol 2007; 19: 142-149.

46. Kirov SA, Harris KM. Dendrites are more spiny on mature hippocampal neurons when synapses are inactivated. Nat Neurosci 1999; 2: 878-883.

cc) (i) $(-) \Theta$ Cell Death and Disease is an open-access journal published by Nature Publishing Group. This work is licensed under a Creative Commons Attribution-NonCommercialNoDerivs 3.0 Unported License. To view a copy of this license, visit http://creativecommons.org/licenses/by-nc-nd/3.0/ 